\title{
ON HERMITE-HADAMARD TYPE INEQUALITIES FOR $F$-CONVEX FUNCTIONS
}

\section{Miroseaw AdAmeK}

Abstract. In this paper we give two different Hermite-Hadamard type inequalities for $F$-convex functions. As special cases of it we get known and new Hermite-Hadamard type inequalities for different concepts of convexity. Mathematics subject classification (2010): 26D15, 26A51, 39B62.

Keywords and phrases: F-convex functions, generalized convexity, Hermite-Hadamard type inequalities.

\section{REFERENCES}

[1] M. AdAMEK, On a problem connected with strongly convex functions, Math. Inequal. Appl. 19, 4 (2016), 1287-1293.

[2] M. ADAMEK, On Jensen type inequalities for $F$-convex functions, accepted for publication in Math. Inequal. Appl.

[3] M. AdAmeK, Remark on F-convex functions, J. Convex Analysis 20, 1 (2021).

[4] G. Alberti, L. Ambrosio, P. Cannarsa, On the singularities of convex functions, Manuscripta Math., 76 (1992), 421-435.

[5] S.S. Dragomir, C.E.M. Pearce, Selected Topics on Hermite-Hadamard Inequalities and Applications, RGMIA Monographs, Victoria University (2002) (https://rgmia.vu.edu.au/monographs/).

[6] A. EL. FARISSI, Simple proof and refinement of Hermite-Hadamard inequality, J. Math. Inequal., 4, 3 (2010), 365-369.

[7] J. HADAMARD, Étude sur les propriétés des fonctions entiéres et en particulier d'une fonction considérée par Riemann, J. Math Pures Appl., 58 (1893), 171-215.

[8] M. KuCZMA, An Introduction to the Theory of Functional Equations and Inequalities. Cauchy's Equation and Jensen's Inequality, PWN-Uniwersytet Śląski, Warszawa-Katowice-Kraków, 1985, 2nd Edition: Birkhäuser, Basel-Boston-Berlin, 2009.

[9] D.S. Mitrinović, I. B. Lacković, Hermite and convexity, Aequat. Math. 28 (1985), 229-232.

[10] N. Merentes, K. Nikodem, Remarks on strongly convex functions, Aequat. Math. 80 (2010), $193-$ 199.

[11] H.V. Ngai, D.T. LuC, M. THÉRA, Approximate convex functions, J. Nonlinear and Convex Anal., 1, 2 (2000), 155-176.

[12] C.P. Niculescu, L.E. Persson, Convex Functions and Their Applications. A Contemporary Approach, Second Edition, CMS Bokks in Mathematics, Springer, 2018, 415+xvii pp.

[13] K. Nikodem, Zs. PÁles, On t-convex functions, Real Anal. Exchange, 29, 1 (2003/2004), 219-228.

[14] K. NikODEM, Zs. PÁLES, Characterizations of inner product spaces by strongly convex functions, Banach J. Math. Anal. 5 (2011), no. 1, 83-87.

[15] Zs. PÁLES, On approximately convex functions, Proc. Amer. Math. Soc., 131, 1 (2003), 243-252.

[16] B.T. POLYAK, Existence theorems and convergence of minimizing sequences in extremum problems with restrictions, Soviet Math. Dokl. 7 (1966), 72-75.

[17] A.W. Roberts, D.E. Varberg, Convex Functions, Academic Press, New York-London, 1973.

[18] J.-P. VIAL, Strong and weak convexity of sets and functions, Math. Oper. Research, 8, 2 (1983), $231-$ 251. 